\title{
Empfehlungen zur Begutachtung von Berufsdermatosen - Erläuterungen anhand von Beispielen aus dem Baugewerbe
}

\section{Skudlik \\ H. J. Schwanitz}

\author{
Guidelines for Medico-Legal Evaluation of Occupational Skin Diseases \\ in Germany - Examples from the Construction Industry
}

\section{Zusammenfassung}

Berufsbedingte Hauterkrankungen sind seit Jahren zahlenmäßig der Spitzenreiter unter den Berufskrankheiten; mehr als ein Viertel aller gemeldeten BK-Verdachtsfälle an die zuständigen Versicherungsträger beziehen sich auf den versicherungsrechtlichen Tatbestand der BK 5101 der BKV. Die gewerbedermatologische Begutachtung zur BK 5101 stellt aufgrund der versicherungsrechtlichen Bedingungen an den Gutachter erhöhte Anforderungen. Um die wesentlichen Aspekte der Qualität berufsdermatologischer Gutachten sowie eine weitestgehende Gleichbehandlung der zu Begutachtenden zu gewährleisten, wurden entsprechende Empfehlungen erarbeitet. Diese werden im vorliegenden Beitrag beispielhaft anhand von Fallkonstellationen aus der Baubranche erläutert.

\section{Abstract}

Occupational skin diseases are the most common occupational diseases in Germany and amount to more than $25 \%$ of all suspected occupational diseases. Medico-legal evaluation of occupational skin diseases necessitate the highest possible quality because of the difficult technical aspects given by the German accident insurance system. To ascertain the quality of medico-legal evaluation and the equal treatment of workers being evaluated specific guidelines for occupational skin diseases were developed and seminars for specialists were initiated. We introduce these guidelines using examples from the construction industry.

\section{Einleitung}

Die Verdachtsanzeigen auf Vorliegen eines Erkrankungsgeschehens im Sinne der BK-Ziffer 5101 der BKV haben in den letzten Jahrzehnten eine stetige Zunahme aufgewiesen. So wurden 19606208 Verdachtsfälle auf Vorliegen eines Erkrankungsgeschehens im Sinne der BK-Ziffer 5101 der BKV an die zuständigen Unfallversicherungsträger gemeldet, 198010931 Verdachtsfälle, 199018717 Verdachtsfälle und 199919458 Verdachtsfälle (dies entspricht für das Jahr 1999 einem Anteil von 26,8\% an allen gemeldeten Berufserkrankungen) [1]. Die BK 5101 führt hiermit in den letzten Jahren die Rangliste der verschiedenen verdachtweise gemeldeten Berufskrankheiten an.
Insgesamt wurden im Jahre 19991503 Berufserkrankungen nach Ziffer 5101 der BKV anerkannt, hieraus ergaben sich in 467 Fällen neue BK-Renten [1].

Die gewerbedermatologische Begutachtung zur BK-Ziffer 5101 der BKV stellt an den Gutachter erhöhte Anforderungen aufgrund der Voraussetzungen im Hinblick auf die „Schwere“, „wiederholte Rückfälligkeit“ und ganz besonders den „objektiven Zwang“ zur Unterlassung der schädigenden Tätigkeit [2].

Um die wesentlichen Aspekte der Qualität berufsdermatologischer Gutachten (Informationserfassung, Informationsverdichtung, Informationsverknüpfung) zu gewährleisten, hat die Ar- 
beitsgemeinschaft für Berufs- und Umweltdermatologie in der Deutschen Dermatologischen Gesellschaft (ABD) mit ihrer Arbeitsgruppe "Qualitätssicherung im BK-Verfahren“ gezielt an entsprechenden Begutachtungsempfehlungen gearbeitet. Diese Empfehlungen wurden am 20.9.1998 vom Grundsatzausschuss „Berufskrankheiten“ des Vorstands des Hauptverbandes der gewerblichen Berufsgenossenschaften beschlossen [3-5].

Des Weiteren wurden von Seiten der Arbeitsgemeinschaft für Berufs- und Umweltdermatologie die Arbeitsgruppen „Bewertung der Allergene bei BK 5101“ [6] und „Erfassung und Bewertung irritativer Hautschäden“ etabliert, u.a. um eine weitestgehende Gleichbehandlung der Versicherten im Hinblick auf die abschließende versicherungsrechtliche Wertung zu gewährleisten.

Ergänzend kann der gewerbedermatologische Gutachter auf Empfehlungen der Deutschen Kontaktallergie-Gruppe (DKG) zur berufsspezifischen Epikutantestung, z.B. bei Maurern und Angehörigen verwandter Berufe [7], zurückgreifen.

\section{Empfehlungen für die Begutachtung bei der BK-Haut} nach [3-5]

\section{Aufbau und Diagnostik}

Gemäß der Empfehlungen sollte das gewerbedermatologische Gutachten einen möglichst einheitlichen Aufbau im Hinblick auf die Wiedergabe der anamnestischen Angaben aufweisen und die Kenntnis des Akteninhaltes widerspiegeln. Der Hautund speziell Handbefund ist exakt dermatologisch zu beschreiben. Die Epikutantestung sollte gemäß der Empfehlungen der Deutschen Kontaktallergie-Gruppe erfolgen [8], unter Berücksichtigung berufsgruppenspezifischer Testempfehlungen [7]. Hierzu sei darauf hingewiesen, dass Berufsstoffe, deren Zusammensetzung nicht genau bekannt ist, im Epikutantest (u. a. aus ethischen Überlegungen) nicht routinemäßig getestet werden sollten. Insbesondere ist die Epikutantestung mit obligat toxischen Berufsstoffen (z.B. zementhaltigen Zubereitungen) obsolet. Ferner sollten Allergene, die in Vortestungen zu sehr starken Reaktionen (dreifach positiv) geführt haben, nur begründet und in höherer Verdünnung exponiert werden. Im Einzelfall kann ein entsprechendes Atopie-Screening mit Pricktestungen bzw. IgEDiagnostik indiziert sein.

Eine umfassende und detaillierte Beschreibung des diagnostizierten Krankheitsbildes ist Voraussetzung für eine nachvollziehbare Zusammenhangsbeurteilung und für die bei der Bescheiderteilung vorzunehmende Differenzierung.

Dies gilt auch für alle sonstigen Befunde, die für die Beurteilung der Genese der Hauterkrankung relevant sein können.

Hervorzuheben ist hierbei die Bedeutung einer sorgfältigen Differenzierung des Krankheitsbildes bei Kontaktekzemen. Eine eingehende Auseinandersetzung mit der Frage, ob es sich im Einzelfall tatsächlich um ein allergisches Kontaktekzem oder um ein Zusammentreffen eines Abnutzungsekzems mit einer bisher klinisch nicht relevanten Berufsstoff-Sensibilisierung handelt, ist ggf. unerlässlich.

\section{Beispiel}

a) 45-jähriger Maurer, seit einem Jahr streng arbeitsabhängige Hauterscheinungen der Hände mit Rötung, Bläschenbildung und Rissbildung an den Fingern beuge- und streckseitig, den Handrücken und den Handinnenflächen mit Streuung auf die Unterarme sowie nachgewiesener Typ-IV-Sensibilisierung gegenüber Kaliumdichromat.

Diagnose: Allergisches Kontaktekzem bei Typ-IV-Sensibilisierung gegenüber Kaliumdichromat.

b) 32-jähriger Fliesenleger, seit über 10 Jahren überwiegend arbeitsabhängige Rötungen und Schuppungen im Bereich sämtlicher Fingerzwischenräume mit gelegentlichem Übergang auf die Handrücken, verstärkt in den Wintermonaten und nach erhöhter Feuchtarbeit (Verfugen, Abwaschen der Fliesen). Handinnenflächen, Fingerbeugeseiten sowie medialer und proximaler Anteil der Handrücken stets erscheinungsfrei, keine Streureaktionen. Nachgewiesene Typ-IV-Sensibilisierung gegenüber Kaliumdichromat. Rhinokonjunctivitis allergica saisonalis. Beugenekzeme des Sohnes.

Diagnose: Kumulativ-subtoxisches Handekzem bei atopischer Disposition.

Auffälliger Befund: berufsbedingte Typ-IV-Sensibilisierung gegenüber Kaliumdichromat.

\section{Ursachenzusammenhang}

Für den Nachweis des Krankheitsbildes ist eine an Sicherheit grenzende Wahrscheinlichkeit erforderlich (sog. Vollbeweis). Insbesondere muss mit an Sicherheit grenzender Wahrscheinlichkeit (Vollbeweis) feststehen, ob und welchen zur Verursachung einer Hauterkrankung geeigneten Einwirkungen der Versicherte über welche Zeiträume ausgesetzt war.

Für die Bejahung des Ursachenzusammenhanges zwischen einer nachgewiesenen hautschädigenden Einwirkung (Abb.1 u.2) und der Hauterkrankung genügt es, dass ein solcher wahrscheinlich ist. Dies ist der Fall, wenn den für einen Zusammenhang sprechenden Umständen das Übergewicht zukommt. Von der Wahrscheinlichkeit eines Ursachenzusammenhangs ist die bloße Möglichkeit zu unterscheiden. Vom Gutachter wird eine Würdigung des Erkrankungsverlaufes dahingehend verlangt, ob ein Ursachenzusammenhang im Sinne der Entstehung oder der Verschlimmerung infrage kommt [2,3] (Tab.1).

Bei der Verschlimmerung ist für die Beurteilung des haftungsausfüllenden Ursachenzusammenhangs zu unterscheiden zwischen vorübergehender Verschlimmerung, die zum Zeitpunkt der Untersuchung wieder abgeklungen ist, und noch andauernden Verschlimmerungen. Nach dem Schweregrad sind abgrenzbare und richtunggebende Verschlimmerungen zu unterscheiden.

\section{Schwere/wiederholte Rückfälligkeit}

Nach ggf. Sicherung eines Ursachenzusammenhanges im o.g. Sinne ist zu klären, ob eine schwere oder wiederholt rückfällige Hauterkrankung im versicherungsrechtlichen Sinne vorliegt. Es reicht aus, wenn die Erkrankung schwer oder wiederholt rückfällig gewesen ist (Tab. 2). 

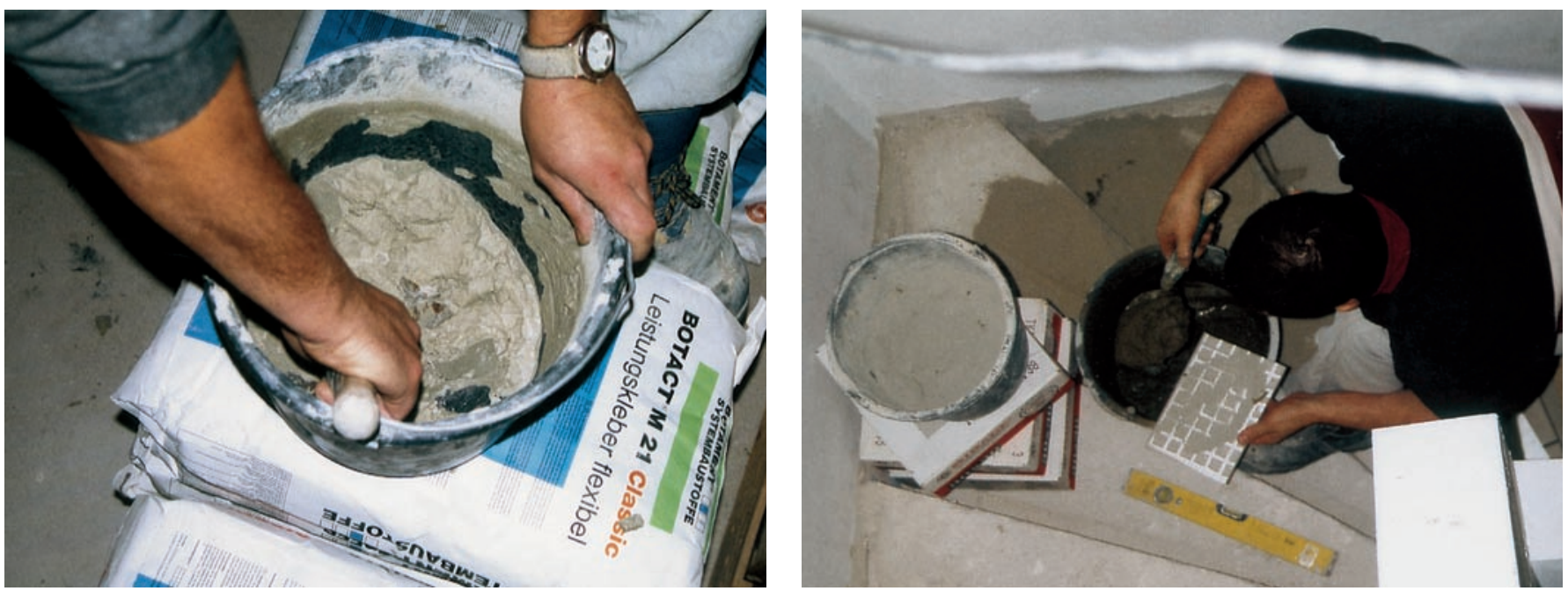

Abb. 1 u. 2 Umgang mit hautgefährdenden Berufssubstanzen im Baugewerbe ohne Hautschutzmaßnahmen.

Tab. 1 Ursachenzusammenhang

\section{Ursachen- $\quad$ Fallgruppe \\ zusammenhang}

Entstehung Toxisch-degenerative (kumulativ-subtoxische) Ekzeme, sofern berufsspezifische Einwirkungen erstmals zur Manifestation geführt haben.

Allergische Kontaktekzeme, sofern die Sensibilisierung beruflich erworben wurde.

Allergische Kontaktekzeme, verursacht durch Allergenkontakte am Arbeitsplatz, auch wenn die Sensibilisierung außerberuflich erworben wurde (zu entschädigen sind nur die durch berufliche Einflüsse entstandenen Hauterscheinungen, nicht die vorbestehende Sensibilisierung). Atopische (Hand-)Ekzeme, die erstmals während einer Berufskrankheit aufgetreten und mit Wahrscheinlichkeit (mit-)ursächlich auf diese zurückzuführen sind (zu entschädigen ist nicht die vorberufliche vorhandene atopische Hautdisposition).

Verschlimmerung Wesentliche Verstärkung vorberuflich festgestellter Hauterscheinungen (z. B. atopisches Handekzem) durch berufsbedingte toxisch-degenerative (kumulativ-subtoxische) Einwirkungen.

Wesentliche Verstärkung eines vorbestehenden, außerberuflich erworbenen, allergischen Kontaktekzems durch berufsbedingten erneuten Kontakt zu demselben Allergen.
Tab. 2 Schwere/wiederholte Rückfälligkeit

\begin{tabular}{|ll}
\hline Schwere & $\begin{array}{l}\text { klinisches Bild (z. B. bedingt durch Zeichen der Super- } \\
\text { infektion oder tiefe Rhagaden usw.) }\end{array}$ \\
& $\begin{array}{l}\text { Ausdehnung (z. B. streuendes allergisches Kontaktekzem, } \\
\text { aerogenes allergisches Kontaktekzem) }\end{array}$ \\
& $\begin{array}{l}\text { Verlauf (z. B. bedingt durch eine schlechte Heilungstendenz } \\
\text { trotz intensivierter therapeutischer Bemühungen) }\end{array}$ \\
& $\begin{array}{l}\text { Dauer (als Zeitfaktor werden hier mindestens } 6 \text { Monate } \\
\text { zugrundegelegt, wobei ärztlicherseits eine ununterbrochene } \\
\text { Behandlungsbedürftigkeit objektiviert sein sollte) }\end{array}$ \\
\hline wiederholte & $\begin{array}{l}\text { drei gleichartige Krankheitsschübe, dazwischen je eine } \\
\text { Rückfälligkeit }\end{array}$ \\
& $\begin{array}{l}\text { Phase mit Abheilung der Hauterscheinungen bzw. Nicht- } \\
\text { Behandlungsbedürftigkeit }\end{array}$ \\
\hline
\end{tabular}

\section{Beispiel}

55-jähriger Bauhelfer, Aufgabe der beruflichen Tätigkeit aufgrund anamnestisch ausgeprägter Handekzeme, welche über ca. 10 Jahre eine arbeitsabhängige Progredienz aufgewiesen hätten. In der Akte sind lediglich zweimalige hausärztliche Behandlungsphasen über jeweils ca. 2 Wochen unter den Diagnosen „Handekzeme“ mit Besserung unter Arbeitsunfähigkeit und externer Kortisonbehandlung dokumentiert. Zum Zeitpunkt einer aktuellen gewerbedermatologischen Begutachtung vier Monate nach Aufgabe der Tätigkeit bis auf Lichenifikation des Hautorganes im Bereich der Hände keine pathologischen Hauterscheinungen, einfach positive Reaktion nach 72 Stunden im Epikutantest gegenüber Kaliumdichromat.

Diagnose: Zustand nach berufsbedingtem Kontaktekzem der Hände, z.B. kumulativ-subtoxisch und/oder allergisch bei berufsbedingter Typ-IV-Sensibilisierung gegenüber Kaliumdichromat.

Ein Ursachenzusammenhang im Sinne der Entstehung ist wahrscheinlich, eine schwere oder wiederholt rückfällige Hauterkrankung kann jedoch unter Würdigung der (spärlichen) Dokumentation nicht konstatiert werden.

\section{Unterlassungszwang}

Für den Fall, dass das Vorliegen einer schweren und/oder wiederholt rückfälligen Hauterkrankung im versicherungsrechtlichen Sinne festgestellt werden kann, ist zu prüfen, ob der objektive Zwang zur Unterlassung der schädigenden Tätigkeit vorliegt. Hierbei ist es nicht erforderlich, dass der Versicherte wegen der Hauterkrankung seine bisherige Beschäftigung völlig aufgeben muss. Vielmehr reicht ein Zwang zum Unterlassen von bestimmten Tätigkeiten aus. Die aufzugebenden Tätigkeiten müssen dem bisher ausgeübten Beruf auch nicht das bestimmende Gepräge geben. Ein Zwang zum Unterlassen der bisher ausgeübten, hautbelastenden Tätigkeit ist jedoch nur zu bejahen, wenn andere Möglichkeiten der Abhilfe nicht ausreichen oder nicht realisierbar sind (Tab.3). Die gutachterliche Beurteilung der o.g. Maßnahmen hat unabhängig davon zu erfolgen, ob der Versicherte die hautbelastende Tätigkeit ggf. bereits aufgegeben hat. 
technische/organisatorische Änderungen am Arbeitsplatz

Anwendung persönlicher Schutzmaßnahmen

Beratung/Schulung über hautschonende Arbeitsweisen

konsequente Ausschöpfung der zur Verfügung stehenden (ambulanten und/oder stationären) medizinischen Optionen

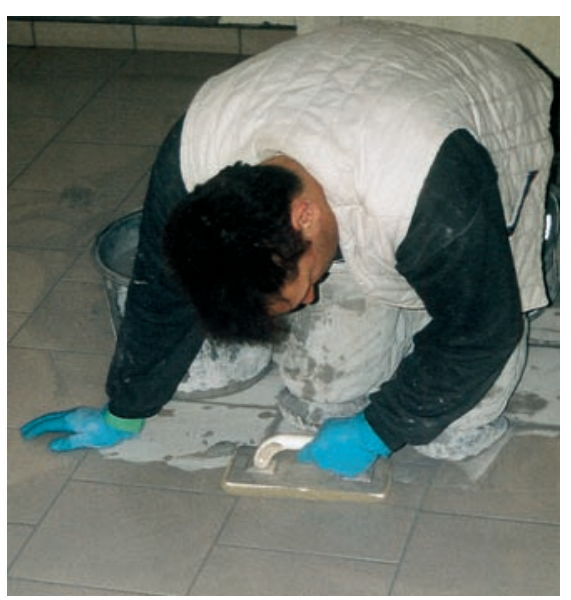

Abb. 3 Anwendung adäquater Hautschutzmaßnahmen: Fliesenleger

Diesbezüglich sollten dem gewerbedermatologischen Gutachter die z.B. bei Berufsdermatosen in Feuchtberufen zur Verfügung stehenden etablierten, hierarchisch gegliederten Präventionskonzepte bekannt sein (Abb. 3) [9].

\section{Beispiel}

a) 38-jähriger Maurer, seit 3 Jahren arbeitsabhängig progrediente entzündliche Hauterscheinungen an den Händen beuge- und streckseitig mit Übergang auf die distalen Unterarme. Seit 2 Jahren unregelmäßige dermatologische Konsultationen, hierbei Dokumentation von Handekzemen u. a. mit Rhagaden und Superinfektion. Nachgewiesene Typ-IV-Sensibilisierungen im Epikutantest gegenüber Kaliumdichromat ( $72 \mathrm{~h}:+$ ), Kobalt ( $72 \mathrm{~h}:+$ ) und Thiuram-Mix ( $72 \mathrm{~h}:+++$ ) sowie einen am Arbeitsplatz verwendeten Thiuram- und Dithiocarbamat-haltigen Schutzhandschuh (72 h: ++).

Diagnose: Berufsbedingtes allergisches Kontaktekzem bei TypIV-Sensibilisierungen gegenüber Kaliumdichromat, Kobalt und Thiuram-Mix.

Versicherungsrechtlich besteht ein Ursachenzusammenhang im Sinne der Entstehung, ferner ist hautärztlicherseits eine schwere Hauterkrankung im versicherungsrechtlichen Sinne dokumentiert.

Es besteht jedoch nicht der objektive Zwang zur Unterlassung der schädigenden Tätigkeit, da noch nicht die entsprechenden Abhilfemaßnahmen gemäß §3 ausgeschöpft wurden.

Im vorliegenden Fall sind hierbei im Rahmen der sekundären Prävention u.a. angezeigt:
Gewährleistung der konsequenten Umsetzung der Vorgaben der technischen Regel für Gefahrstoffe (TRGS) 613 („Ersatzstoffe, Ersatzverfahren und Verwendungsbeschränkungen für chromathaltige Zemente und chromathaltige zementhaltige Zubereitungen“), soweit noch nicht im Rahmen der primären Prävention umgesetzt [10].

Im Hinblick auf persönliche Schutzmaßnahmen die Zurverfügungstellung entsprechender Hautschutzexterna sowie insbesondere thiuramfreier (und aufgrund häufiger Kreuzreaktionen dithiocarbamatfreier) adäquater Schutzhandschuhe (z.B. thiuramfreie nitrilbeschichtete Baumwollhandschuhe oder PVCHandschuhe).

Durchführung einer engmaschigen stadiengerechten möglichst nicht hautatrophisierend wirkenden ambulanten dermatologischen Therapie im Rahmen des §3.

Sofern sich auch nach Ausschöpfung der o.g. Maßnahmen keine wesentliche Besserung des Verlaufes zeigt, wären darüber hinaus die intensivierten Maßnahmen der tertiären Prävention, z. B. im Rahmen eines stationären Heilverfahrens angezeigt zur Intensivierung der therapeutischen Maßnahmen und Optimierung der Hautschutzmaßnahmen, gegebenenfalls mit gesundheitspädagogischer Intervention [11].

b) 24-jähriger Fliesenleger mit ausschließlicher Tätigkeit als sogenannter „Säurefliesner“ in Laboren und technischen Anlagen. Seit ca. 2 Jahren subjektiv geringgradige arbeitsabhängige Hauterscheinungen im Bereich der Fingerzwischenräume, welche bislang nicht zu ärztlichen Konsultationen geführt hatten. Seit 3 Monaten jedoch ausgeprägtere arbeitsabhängige entzündliche Hauterscheinungen im Bereich der Hände sowie arbeitsabhängig flächenhafte entzündliche Hauterscheinungen des Gesichtes mit ausgeprägten Lidödemen. Aufgrund dieser Hauterscheinungen wurde zweimalig hautärztlicherseits eine zwei- bzw. dreiwöchige Arbeitsunfähigkeitszeit attestiert mit jeweils ärztlicherseits dokumentierter Abheilung der entzündlichen Hauterscheinungen am Ende der AU-Zeiten. Es traten jeweils ein bzw. zwei Tage nach Wiederaufnahme der beruflichen Tätigkeit erneut ausgeprägte entzündliche Hauterscheinungen des Gesichtes auf, weshalb zuletzt u.a. systemische Glukokortikosteroide verordnet wurden. Seitdem wurde zunächst von Seiten des betreuenden Hautarztes fortlaufende Arbeitsunfähigkeit attestiert.

In der Epikutantestung nach 72-stündiger Ablesung dreifach positive Reaktion gegenüber Epoxidharz.

\section{Diagnose:}

1. Berufsbedingtes allergisches, teils aerogenes Kontaktekzem der Hände und des Gesichtes bei Typ-IV-Sensibilisierung gegenüber Epoxidharz

2. Verdacht auf Zustand nach berufsbedingtem kumulativ-subtoxischen Handekzem (anamnestisch).

Im Hinblick auf das teils aerogene allergische Kontaktekzem bei Typ-IV-Sensibilisierung gegenüber Epoxidharz besteht ein Ursachenzusammenhang im Sinne der Entstehung, es ist im versicherungsrechtlichen Sinne sowohl eine schwere als auch wiederholt rückfällige Hauterkrankung dokumentiert. 
Der Umgang mit epoxidharzhaltigen Klebern ist für einen Fliesenleger mit der oben genannten Spezialisierung nicht meidbar. Bei Vorliegen eines aerogenen allergischen Kontaktekzems bei Typ-IV-Sensibilisierung gegenüber Epoxidharz sind zudem adäquate Hautschutzmaßnahmen nicht zur Verfügung zu stellen. Daher besteht im vorliegenden Fall objektiv der Zwang zur Unterlassung der schädigenden Tätigkeit [12].

\section{Minderung der Erwerbsfähigkeit}

Sofern die Voraussetzungen zur Anerkennung einer BK nach Ziffer 5101 der BKV vorliegen, erfolgt die Einschätzung der Minderung der Erwerbsfähigkeit auf dem allgemeinen Arbeitsmarkt sowohl anhand der Auswirkungen der berufsbedingten Allergie als auch anhand des Ausmaßes der verbliebenen Hauterscheinungen, auch nach irritativer Schädigung. Hierzu hat die $\mathrm{Ar}$ beitsgemeinschaft für Berufs- und Umweltdermatologie (ABD) in Zusammenarbeit mit dem Hauptverband der gewerblichen Berufsgenossenschaften (HVBG) eine tabellarische Empfehlung zur Einschätzung der MdE auf der Basis langjähriger Erfahrungssätze zusammengestellt [2]. Diese MdE-Tabelle hat die davor gültigen Empfehlungen abgelöst. Dabei ist die frühere Überbetonung verbliebener allergischer Berufskrankheitenfolgen zugunsten einer „paritätischen“ Berücksichtigung von resultierenden irritativen Hautschädigungen korrigiert worden. Höchstrichterlich ist den gültigen MdE-Empfehlungen der $\mathrm{ABD}$ und des HVBG mittlerweile der Rang eines antizipierten Sachverständigengutachtens zuerkannt worden (BSG vom 30.6.1998, B 2 U 41/97 R).

Die mit der neuen MdE-Tabelle erfolgende stärkere Berücksichtigung der kumulativ-subtoxischen/irritativen Schädigungen des Hautorgans trägt sowohl der Bedeutung dieser Einwirkungen für die Pathogenese berufsbedingter Handekzeme als auch dem quantitativen Vorkommen derartiger Noxen auf dem allgemeinen Arbeitsmarkt Rechnung.

Die ermittelten berufsbedingten Sensibilisierungen sind im Hinblick auf die hieraus resultierenden Auswirkungen der Allergien einzuschätzen. Hierbei hat der gewerbedermatologische Gutachter gemäß der aktuell gültigen MdE-Tabelle die Möglichkeit, die Auswirkungen der berufsbedingten Allergie als „geringgradig“, „mittelgradig“ oder „schwerwiegend“ einzuschätzen. Maßgebliche Beurteilungskriterien zur Einordnung in diese drei Gruppen sind neben der Anzahl der zu berücksichtigenden Allergene und deren Sensibilisierungsgrad insbesondere auch die angenommene Verbreitung auf dem allgemeinen Arbeitsmarkt („wenig“ bzw. „gering verbreitet“, „weit verbreitet“ bzw. „sehr weit verbreitet“).

Die Einschätzungen des Verbreitungsgrades der jeweiligen Berufsstoffe differieren, so dass eine versicherungsrechtliche Gleichbehandlung der Versicherten bei gewerbedermatologischer Begutachtung durch verschiedene Gutachter bisher nicht gewährleistet war.

Dies konnte unter anderem auch im Rahmen einer Befragung einer Gruppe erfahrener Berufsdermatologen gezeigt werden: Selbst innerhalb einer Expertengruppe ist die Bewertung für die erfragten 19 häufigen Berufsallergene unterschiedlich [13].
Um eine rationale Beurteilung der „Auswirkung einer Allergie“ bei der BK 5101 der BKV zu ermöglichen, wurden daher von der Arbeitsgruppe „Bewertung der Allergene bei BK 5101“ der Arbeitsgemeinschaft für Berufs- und Umweltdermatologie in der Deutschen Dermatologischen Gesellschaft Vorschläge zur Bewertung der Allergene erarbeitet [6].

Grundlage der erarbeiteten Empfehlungskriterien waren epidemiologische Untersuchungen, allergologische Besonderheiten der jeweiligen Allergene, arbeitstechnische Erkenntnisse sowie die offizielle Beschäftigtenstatistik der Bundesanstalt für Arbeit, aus der errechnet werden konnte, welchen Anteil die verschlossenen Arbeitsfelder am gesamten Arbeitsmarkt haben.

Bei der Bewertung der Auswirkung einer Allergie ist u.a. zu berücksichtigen, dass bei Vorliegen klinisch hochgradiger Sensibilisierungen in manchen Fällen mehr Arbeitsmöglichkeiten verschlossen sind als bei Sensibilisierungen geringerer Intensität. Ferner ist u. a. zu berücksichtigen, dass mehrere Sensibilisierungen nicht einfach addiert werden dürfen, sondern beurteilt werden muss, welche Arbeitsfelder insgesamt aufgrund der berufsbedingten Sensibilisierungen verschlossen sind. Diese grundsätzlichen Einschätzungen wurden jüngst auch in aktuellen Sozialgerichtsurteilen bestätigt (LSG NRW vom 28.3.2001, L 17 U 289/99, SG Hannover vom 28.11. 2002, S 36 U 319/98).

\section{Beispiel}

58-jähriger Maurer mit bereits von Seiten des Unfallversicherungsträgers anerkannter BK nach Ziffer 5101 der BKV aufgrund eines (als BK-Folge anerkannten) „allergischem Kontaktekzems der Hände bei Typ-IV-Sensibilisierung gegenüber Kaliumdichromat und Kobalt“. Die Aufgabe der beruflichen Tätigkeit erfolgte sechs Monate vor einer aktuellen Nachuntersuchung zur Bewertung der BK-Folgen.

Anamnestisch sei eine mäßige erhöhte Empfindlichkeit der Handrücken verblieben, ferner würden sich insbesondere in den Sommermonaten bzw. bei starkem Schwitzen und nach dem Tragen (chromatgegerbter) Lederschuhe entzündliche Reaktionen im Bereich der Fußrücken zeigen. Hautärztlicherseits ist einmalig in den Sommermonaten nach Aufgabe der beruflichen Tätigkeit ein mäßiggradiges Ekzem der Fußrücken dokumentiert.

Klinisch zeigt sich zum Zeitpunkt der Nachuntersuchung im Bereich der Handrücken eine Lichenifikation des Hautorganes. Hautphysiologisch lässt sich mittels einer differenziellen Irritationstestung [14] im Bereich der Handrücken eine verbliebene irritative Schädigung des Hautorganes objektivieren. Bei bereits nach Aktenlage gesicherter berufsbedingter Typ-IV-Sensibilisierung gegenüber Kaliumdichromat und Kobalt zeigen sich in der Titrationstestung beider Allergene Hinweise für das Vorliegen entsprechend hochgradiger Sensibilisierungen (Abb.4), zudem ergaben sich auch anamnestisch Hinweise auf eine klinisch hochgradige Dichromat-Sensibilisierung (s. o.: Fußrücken).

Hieraus ergeben sich zusammenfassend schwerwiegende Auswirkungen der berufsbedingten Allergie sowie leichtgradige Auswirkungen der verbliebenen irritativen Hautschädigung. Beispielhaft sind in Tab. 4 die zu berücksichtigenden Aspekte bei der 


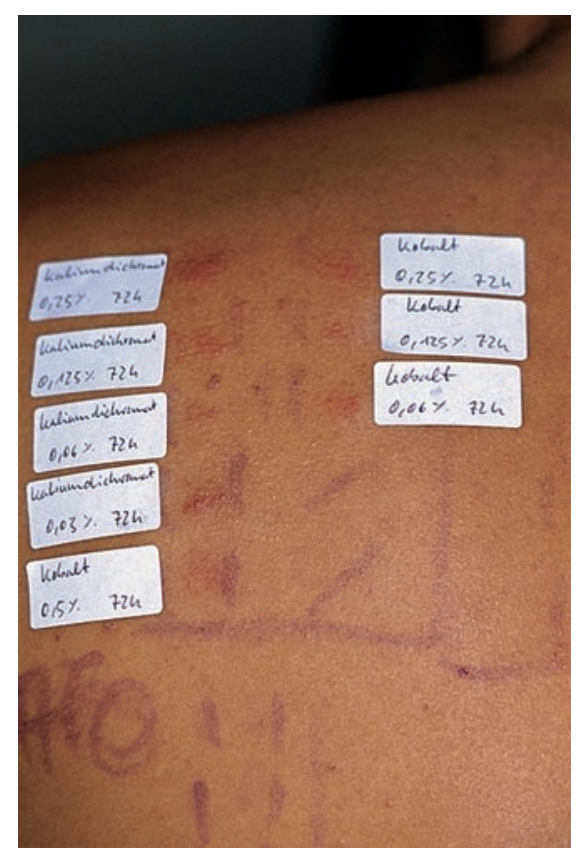

Abb. 4 Epikutantestung: Titration von Kaliumdichromat und Kobalt mit positiven Reaktionen auch auf höhere Verdünnungen. versicherungsrechtlichen Wertung einer berufsbedingten Kaliumdichromat-Sensibilisierung aufgeführt.

Es resultiert unter Zugrundelegung der aktuell gültigen MdE-Tabelle der Arbeitsgemeinschaft für Berufs- und Umweltdermatologie eine MdE von 20\% (Tab. 5).

\section{Qualitätssicherung}

Von der Arbeitsgemeinschaft für Berufs- und Umweltdermatologie wurden zur Gewährleistung einer Qualitätssicherung der berufsdermatologischen Begutachtung u.a. drei ganztägige interdisziplinäre Fortbildungsseminare konzipiert, die von erfahrenen Berufsdermatologen und juristisch versierten Verwaltungsvertretern durchgeführt werden. Nach Absolvierung aller drei Seminare und der Erfüllung weiterer Kriterien wird von der Arbeitsgemeinschaft für Berufs- und Umweltdermatologie ein Zertifikat „Berufsdermatologie“ ausgestellt, so dass der Teilnehmer potenziellen Gutachtenauftraggebern besonderes berufsdermatologisches Engagement und berufsdermatologische Kompetenz nachweisen kann [15].

\section{Literatur}

Tab. 4 Versicherungsrechtliche Wertung der berufsbedingten Kaliumdichromat-Sensibilisierung [nach 6]

\begin{tabular}{|c|c|}
\hline \multirow[t]{2}{*}{ verschlossene Berufsgruppen } & $\begin{array}{l}\text { Betonbauer, Farben- und Lackhersteller, } \\
\text { Färber, Feuerwerksartikelhersteller, } \\
\text { Fliesenleger, Galvaniseure, Gerber, Graveure, } \\
\text { Holzarbeiter, Keramikhersteller, Leder- } \\
\text { verarbeiter, Maurer, Metallarbeiter, Pelz- } \\
\text { bearbeiter, Schweißer, Tonbandhersteller } \\
\text { und Verchromer }\end{array}$ \\
\hline & $\begin{array}{l}\text { Cave: Sensibilisierung auch möglich durch } \\
\text { das Tragen chromgegerbter Lederhandschuhe } \\
\text { oder Sicherheitsschuhe }\end{array}$ \\
\hline Auswirkung der Allergie & mittelgradig bis schwerwiegend \\
\hline \multirow{5}{*}{$\begin{array}{l}\text { Kriterien „schwerwiegender } \\
\text { Auswirkungen“ }\end{array}$} & klinisch hochgradige Sensibilisierung \\
\hline & $\begin{array}{l}\text { Hinweise für eine hochgradige Sensibilisie- } \\
\text { rung (evtl. gestützt durch Epikutantestung } \\
\text { mit einer Verdünnungsreihe): }\end{array}$ \\
\hline & $\begin{array}{l}\text { Streureaktionen des allergischen Kontakt- } \\
\text { ekzems }\end{array}$ \\
\hline & Kontaktekzem nach aerogener Exposition \\
\hline & $\begin{array}{l}\text { Unverträglichkeit von ledernen Schuhen oder } \\
\text { Handschuhen }\end{array}$ \\
\hline
\end{tabular}

Tab. 5 MdE-Tabelle, Anwendung bezogen auf ein Fallbeispiel (Auswirkung der Allergie: schwerwiegend, Ausmaß der Hauterscheinungen: leicht). Gemeinsame Empfehlungen der ABD und des HVBG

\begin{tabular}{lcccc|}
\hline $\begin{array}{l}\text { Auswirkung } \\
\text { der Allergie }\end{array}$ & $\begin{array}{l}\text { Hauterscheinungen/Auswirkungen der irritativen Schädigung } \\
\text { keine }\end{array}$ & $\begin{array}{c}\text { leicht } \\
\text { mittel }\end{array}$ & schwer \\
\hline keine & $0 \%$ & $10 \%$ & $20 \%$ & $25 \%$ \\
\hline geringgradig & $0 \%$ & $10 \%$ & $20 \%$ & $25 \%$ \\
\hline mittelgradig & $10 \%$ & $15 \%$ & $25 \%$ & $30 \%$ \\
\hline schwerwiegend & $20 \%$ & $\mathbf{2 0} \%$ & $30 \%$ & $\geq 30 \%$ \\
\hline
\end{tabular}

${ }^{1}$ Plinske W, Drexel G, Drechsel-Schlund C, Butz M. BK-DOK '99 - Dokumentation des Berufskrankheiten-Geschehens in Deutschland. Hauptverband der gewerblichen Berufsgenossenschaften (HVBG). St. Augustin: Plump OHG-Rheinbreitbach, 2001 krankheit - Rechtliche und medizinische Grundlagen für Gutachter, Sozialverwaltung, Berater und Gerichte. 6. Aufl. Berlin: Erich Schmidt Verlag, 1998

${ }^{3}$ Brandenburg S, Schwanitz HJ, John SM. Empfehlungen für die Begutachtung von Berufskrankheiten nach BK 5101. Dermatosen 1999; 47: $109-114$

${ }^{4}$ Schwanitz HJ, John SM, Brandenburg S. Empfehlungen für die Diagnostik von Berufskrankheiten nach BK 5101. Dermatosen 1998; 46: $253-260$

${ }^{5}$ Schwanitz HJ. Begutachtung berufsbedingter Hauterkrankungen aus Sicht des Dermatologen und Allergologen. In: Schwanitz HJ, Szliska C (Hrsg.). Berufsdermatosen. München-Deisenhofen: Dustri-Verlag, 2001

${ }^{6}$ Diepgen TL, Dickel H, Becker D, Blome O, Geier J, Schmidt A, Schwanitz HJ, Skudlik C, Wagner E für die Arbeitsgruppe „Bewertung der Allergene bei BK 5101“ der Arbeitsgemeinschaft für Berufs- und Umweltdermatologie in der Deutschen Dermatologischen Gesellschaft. Beurteilung der Auswirkung von Allergien bei der Minderung der Erwerbsfähigkeit im Rahmen der BK 5101. Teil I: Acrylate/Methacrylate, Epoxidharz-Systeme, Formaldehyd, Dichromat, Kolophonium, Latex, Nickel, p-Phenylendiamin. Dermatol Beruf Umwelt 2002; 50: 139-154

${ }^{7}$ Geier J, Brehler R, Eck E, Koch P, Peters KP, Rakoski J, Rothe A, Schnuch A, Szliska C, Uter W. Berufsspezifische Epikutantestung bei Maurern und Angehörigen verwandter Berufe - Empfehlungen der Arbeitsgruppe „Berufs-Testreihen“ der Deutschen Kontaktallergie-Gruppe. Dermatosen 1999; 47: 29-33

${ }^{8}$ Schnuch A, Aberer W, Agathos M, Brasch J, Frosch PJ, Fuchs T, Richter G. Epikutantest. In: Korting HC, Callies R, Reusch M, Schlaeger M, Schöpf E, Sterry W (Hrsg). Dermatologische Qualitätssicherung - Leitlinien und Empfehlungen. 2. Aufl. Germering: Zuckschwerdt-Verlag, 2001

9 Skudlik C, Schwanitz HJ. Berufskrankheiten der Haut. Trauma Berufskrankh 2002; 4: 151 - 162

10 Struppek K, Ludwig S. Prävention des Chromat-Ekzems im Baugewerbe. Dermatosen 1999; 47: 13-15

${ }^{11}$ Schwanitz HJ. Tertiäre Prävention von Berufsdermatosen - Bericht über das Forschungsprojekt „Stationäre Präventionsmaßnahme für hautkranke Versicherte“. Dermatol Beruf Umwelt 2002; 50: 212 - 217
2 Schönberger A, Mehrtens G, Valentin H. Arbeitsunfall und Berufs- 
${ }^{12}$ Skudlik C, Schwanitz HJ. Sensibilisierung gegen ubiquitäre Allergene: Problematik der Prävention und der versicherungsrechtlichen Wertung, aufgezeigt an Fallbeispielen. Essen: 1. Essener Dermatologischbetriebsärztliches Symposium, 24.4.2002

${ }^{13}$ Richter G. Minderung der Erwerbsfähigkeit durch Allergien bei der BK 5101 - Analyse einer Expertenbefragung. Dermatosen 1994; 42: $138-142$

${ }^{14}$ John SM. Klinische und experimentelle Untersuchungen zur Diagnostik in der Berufsdermatologie. Konzeption einer wissenschaftlich begründeten Qualitätssicherung in der sozialmedizinischen Begutachtung. Osnabrück: Universitätsverlag Rasch, 2001

${ }^{15}$ John SM, Blome O, Brandenburg S, Wehrmann W, Schwanitz HJ. Qualitätssicherung in der Berufsdermatologischen Begutachtung: Curriculum der Gutachten-Seminare der Arbeitsgemeinschaft für Berufsund Umweltdermatologie in Zusammenarbeit mit dem Hauptverband der gewerblichen Berufsgenossenschaften und dem Berufsverband der Deutschen Dermatologen. Dermatol Beruf Umwelt 2001; 49: $155-160$

\section{Buchbesprechung}

\section{Haut und Seele: Schönheit und Wohlbefinden durch inneres Gleichgewicht}

F. Gschnait, W. Exel

Wien: Carl Ueberreuter, 2002. 252 S., Geb. 24,90 .

ISBN: 3-8000-3838-2

Das ist ein gutes, brauchbares und übersichtliches Buch, das der erfahrene Wiener Dermatologe mit seinem Kollegen, praktischer Arzt und Journalist in fruchtbarer Kombination, hier für Patienten, deren Familien, für Laien also, und auch für die praktisch tätigen Ärzte vorlegt. Es übertrifft vieles, was gleichgesinnten Bemühungen entsprang, durch Vernunft, Praktikabilität und Glaubwürdigkeit.

Es beginnt mit einem kurzen Blick in die mythologischen Ursprünge des Zusammenspiels zwischen Haut und Seele, zeigt die sprichwörtliche Verankerung desselben in den Volksweisheiten und in der Umgangssprache. „Es geht unter die Haut“, das Buch und sein Anliegen im Untertitel zu „Schönheit und Wohlbefinden durch inneres Gleichgewicht“. Angesprochen werden die Haut als Grenzorgan und Spiegel der Seele, wesentliche Hautkrankheiten und deren seelische Komponenten, seelische Probleme für die Haut und die 6 häufigsten Lebenskrisen, mit Hilfen zu deren Bewältigung. Also, einmal von der Haut zur Seele gedacht und gewertet, dann aber auch umgekehrt von der Seele aus betrachtet, die Auswirkungen auf die Haut und den Menschen als Ganzes.

Das Buch ist gut und übersichtlich, einheitlich gegliedert, die einzelnen Kapitel und die Krankheitsbeschreibungen sind immer gleich strukturiert und erleichtern den Zugang, auch graphisch. Falsche Ansichten und Irrmeinungen werden sehr deutlich offengelegt (als sog. „Mythen“ bezeichnet) und die richtige Ansicht jeweils angeschlossen. Dies ist ein besonderer Lichtblick des Buches, das sich zudem sehr leicht liest.

Hocherfreulich ist auch die Offenlegung, dass Menschen, wahrscheinlich kulturhistorisch zu deuten, ausgesprochen geeignete Instrumente und Fähigkeiten haben, Stresssituationen zu bewältigen und sogar biopositiv zu nutzen, während uns die Fähigkeit weitgehend abgeht, Störungen der Rhythmen (Tag-Nacht, Monaten, Jahreszeiten und solche im Lebensablauf der Generationen, etc.) zu bewältigen oder gar zu nutzen. Die daraus bewirkten Hautveränderungen und seelischen Störungen sind dargelegt. Vermissen wird man die Beobachtung, dass bei chronischen Hautkrankheiten (Neurodermitis atropica, Psoriasis vulgaris u.a.) die immer wiederkehrende Herausforderung durch die Erkrankung und deren Bewältigung auch zu einem biopositiven „Schulungseffekt“ führen kann und anderen Lebensbereichen zugute kommt. Dies zu berücksichtigen und einzubauen darf einer, übrigens sehr wünschenswerten, Zweitauflage vorbehalten bleiben. Es ist dieses ein gutes, brauchbares und übersichtliches Buch, das hohe Anerkennung und beste Empfehlung verdient.

E. G. Jung, Heidelberg 